Provided for non-commercial research and educational use only. Not for reproduction or distribution or commercial use.
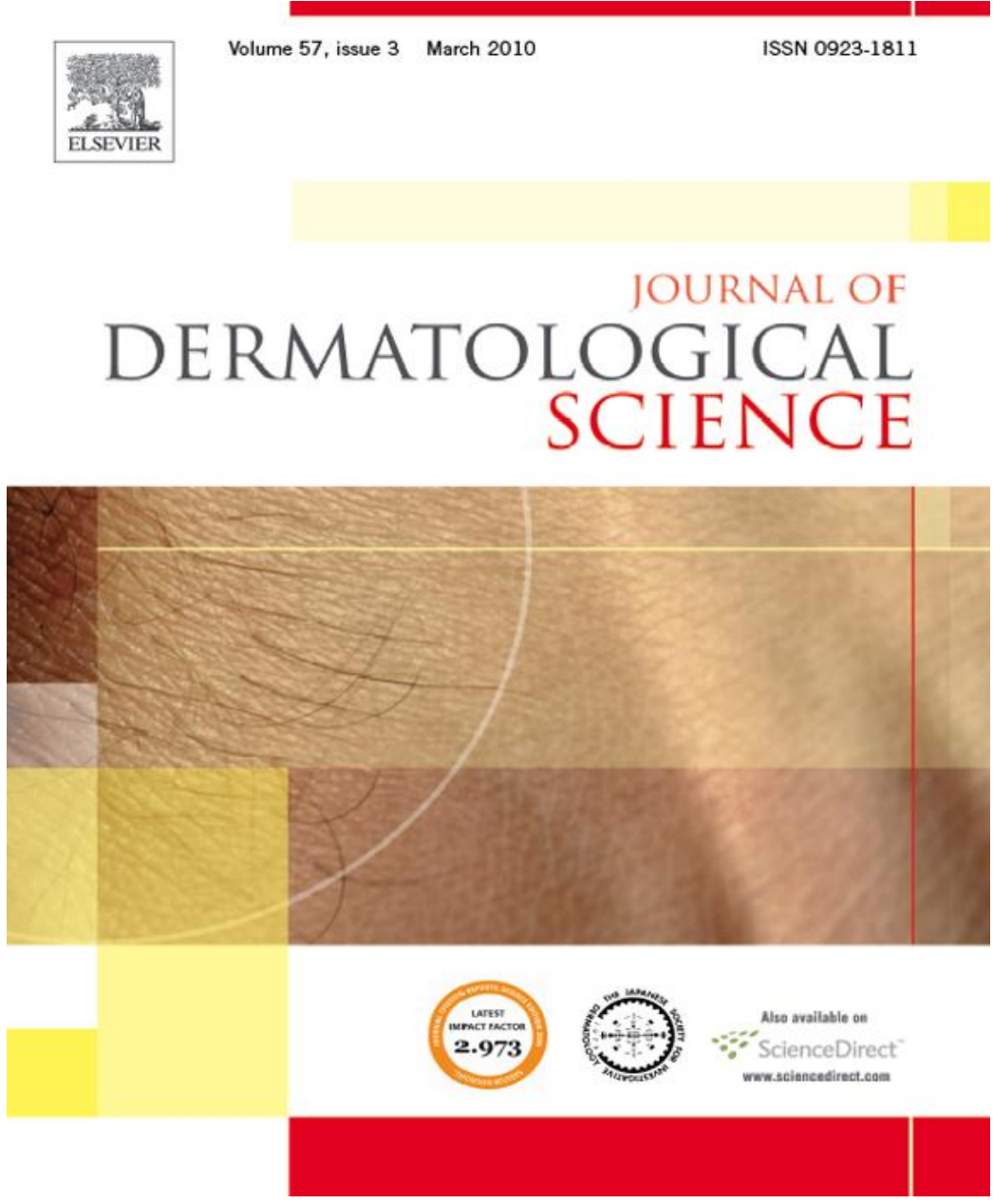

This article was originally published in a journal published by Elsevier, and the attached copy is provided by Elsevier for the author's benefit and for the benefit of the author's institution, for non-commercial research and educational use including without limitation use in instruction at your institution, sending it to specific colleagues that you know, and providing a copy to your institution's administrator.

All other uses, reproduction and distribution, including without limitation commercial reprints, selling or licensing copies or access,

or posting on open internet sites, your personal or institution's website or repository, are prohibited. For exceptions, permission may be sought for such use through Elsevier's permissions site at:

http://www.elsevier.com/locate/permissionusematerial 


\title{
Histological and transcriptional study of angiogenesis and lymphangiogenesis in uninvolved skin, acute pinpoint lesions and established psoriasis plaques: An approach of vascular development chronology in psoriasis
}

\author{
Audrey Henno ${ }^{\mathrm{a}, \mathrm{b}, *}$, Silvia Blacher ${ }^{\mathrm{c}}$, Charles A. Lambert ${ }^{\mathrm{b}}$, Christophe Deroanne ${ }^{\mathrm{b}}$, Agnès Noël ${ }^{\mathrm{c}}$, \\ Charles Lapière $^{\mathrm{b}}$, Michel de la Brassinne ${ }^{\mathrm{a}}$, Betty V. Nusgens ${ }^{\mathrm{b}}$, Alain Colige ${ }^{\mathrm{b}}$ \\ ${ }^{a}$ Department of Dermatology, University Hospital of Liège, Belgium \\ ${ }^{\mathrm{b}}$ Laboratory of Connective Tissues Biology, GIGA-R, 4000 Liege, Belgium \\ ${ }^{\mathrm{c}}$ Laboratory of Tumor and Development Biology, GIGA-R, University of Liège B23, 4000 Liège, Belgium
}

\section{A R T I C L E I N F O}

\section{Article history:}

Received 30 July 2009

Received in revised form 3 December 2009

Accepted 9 December 2009

\section{Keywords:}

Psoriasis

Angiogenesis

Lymphangiogenesis
A B S T R A C T
Background: Dysregulation of angiogenesis and lymphangiogenesis could participate in psoriasis
pathogenesis. Analysis of nascent psoriasis lesions should help at identifying early vascular anomalies.
Objective: To analyse vascular development, angiogenesis and lymphangiogenesis markers expression in
uninvolved skin in psoriatic patients (N), early psoriasis lesions or pinpoints (PP) and psoriasis plaques (PSO).
Methods: Skin biopsies were taken in 17 patients in N and in PSO and/or PP. The mRNA steady-state level
of angiogenesis and lymphangiogenesis markers was measured by RT-PCR. Immunohistochemistry was
performed for von Willebrand factor, podoplanin, Ki-67 and VEGFR3. Blood (BV) and lymphatic (LV)
vessels expansion was measured by computer-assisted morphometry.
Results: Clinical and epidermal aspects indicated that PP are intermediate between N and PSO. While
total BV area was already increased in PP similarly to PSO as compared to N, LV area in PP was
intermediate between N and PSO. Mean LV size was identical in N and PP and increased in PSO, mean BV
size in PP being intermediate between N and PSO. VEGF-A 189 variant was increased in PP as compared
to N and PSO. As compared to N, angiogenesis markers (VEGF-A isoforms, PlGF, VEGFR2, NRP-1), VEGF-C
and NRP-2 were similarly increased in PP and PSO. Keratin 16 and the lymphangiogenesis markers
(VEGFR3, prox-1) were intermediate in PP.
Conclusion: These data suggest that the expansion of lymphatic vessels occurs after blood vascular
development in psoriasis. Expansion of BV in PP could be followed by vessel enlargement during
progression to PSO, in parallel with a decreased VEGF-A $189 /$ VEGF-A 121 balance in plaques.
C 2010 Japanese Society for Investigative Dermatology. Published by Elsevier Ireland Ltd. All rights reserved.

\section{Introduction}

Psoriasis, a common inflammatory disease of the skin affecting $2-3 \%$ of the European population, is in part genetically determined [1]. Histological features of psoriasis include epidermal hyperplasia, impaired epidermal differentiation, accumulation of inflammatory cells and excessive angiogenesis with enlarged, tortuous and hyperpermeable dermal blood vessels [2]. One of the earliest events in the development of psoriatic plaques is a vascular network expansion, which occurs before epidermal changes $[3,4]$ and persists after clearance of the clinical lesions [5].

\footnotetext{
* Corresponding author at: Laboratory of Connective Tissues Biology, Centre Hospitalier Universitaire de Liège, B 23, B-4000 Sart Tilman, Belgium. Tel.: +32 43662469; fax: +32 43662457.

E-mail address: audrey.henno@gmail.com (A. Henno).
}

Among the numerous mediators governing vascular expansion [6], vascular endothelial growth factors (VEGF) family largely participates in the development of the skin lesions in psoriasis [7]. VEGF-A induces blood endothelial cell (EC) proliferation and increased vascular permeability by activating two related tyrosinekinase receptors, VEGFR1 and VEGFR2. VEGF-A binding on VEGFR2, which is the major signaling receptor on the surface of EC, is further potentiated by interaction with neuropilin-1 (NRP-1) acting as a co-receptor [8]. In psoriatic lesions, VEGF-A overexpressed in the activated epidermal cells is responsible for the expression of its own receptor-2 in EC [7]. Alternative splicing of the VEGF-A premRNA generates at least six molecular variants that display biological characteristics related to exons combination. The major isoforms are VEGF-165 and VEGF 121. VEGF 206, 189 and, to a lesser extent, VEGF 165 contain heparin-binding domains allowing their sequestration within the extracellular matrix, which can be reversed by proteolysis [8]. VEGF 121 and a new isoform, VEGF 
111 , that is induced by genotoxic agents but not found in normal and psoriasis human skin $[9,10]$, are freely diffusible. Placental growth factor (PlGF) is another important mediator of skin angiogenesis [11] that may directly activate VEGFR1, thus potentiating VEGF activity, and that also promotes bone-marrow-derived precursor cells mobilization and monocyte chemotaxis [11,12]. As for VEGF-A, alternative exon splicing produces four isoforms, PIGF-1 to -4 , that differ by their binding affinities for heparin [13,14]. PlGF-2 is membrane-associated; PlGF-1 is diffusible while PIGF-3 and PlGF-4 are expressed in placenta and umbilical vein EC [14] but not in adult skin [10].

New insights have been obtained into the mechanisms governing lymphatic development and function during the last decade [15]. VEGF-C and -D have been identified as growth factors stimulating lymphangiogenesis [16]. The activation of their tyrosine-kinase receptor VEGFR3 leads to the proliferation of lymphatic EC while targeted deletion of VEGF-C in mice impairs the development of small lymphatic vessels and is lethal [16]. VEGFR3 is expressed in venous as well as lymphatic EC during embryogenesis but only in lymphatic EC after birth except for the fenestrated blood vessels of some organs [17]. Neuropilin-2a (NRP$2 \mathrm{a}$ ) is a receptor for the semaphorins and acts as a co-receptor for VEGF-A and VEGF-C. It enhances some of the biological responses elicited by these growth factors such as EC survival and migration, through interaction with VEGFR2 and VEGFR3 $[18,19]$. Prox-1 is a homeotic protein that specifies the lymphatic cell fate by reprogramming the transcriptome of embryonic venous EC [20]. Little is known about the mechanisms and the chronology of lymphatic development in psoriasis lesions [21,22].

Psoriasis lesions start as erythematous, slightly raised lesions of $0.5 \mathrm{~mm}$ diameter called prepinpoint papules. By lateral growth, they can lead to scaling pinpoint lesions (PP) of several millimeters of width that precede the formation of plaques (PSO) [23]. Investigating these acute psoriatic lesions is thus expected to bring information on the mechanisms initiating the development of the cutaneous alterations [24,25]. Dermal changes are preponderant in early psoriasis lesions and are characterized by papillary oedema, dilation of both superficial blood vessels and lymphatics, and by a perivascular cellular infiltration in the papillary and upper reticular layers [25]. Histochemical studies have shown that these perivascular infiltrates contain monocytes and macrophages, little lymphocytes and a large number of polymorphonuclears $[25,26]$. Epidermal changes comprise mild hyperplasia with slight epidermal parakeratosis and focal loss of the granular layer as well as circumscribed spongiosis.

The aim of our study was to compare blood and lymphatic vascular expansion as well as the expression of several angiogenesis and lymphangiogenesis markers in the uninvolved skin of psoriasis patients $(\mathrm{N})$, in early psoriasis lesions (PP) (acute pinpoint lesions measuring $4 \mathrm{~mm}$ of width among patients with plaque psoriasis) and in psoriatic plaques (PSO).

\section{Materials and methods}

\subsection{Patients (Table 1)}

Adult patients suffering of moderate to severe plaque-type psoriasis and who had not received any systemic treatment for 1 month or any topical therapy for 2 weeks were selected (Table 1 ). Their mean PASI score was 15.8. After local anaesthesia (1\% xylocaïne with adrenalin), 11 patients (mean age: 43 years old, $2 \mathrm{~F} /$ 9M, mean PASI score: 16.5 ) underwent skin punch biopsy (4 mm) in a plaque lesion (PSO) and in the distant uninvolved skin (N). Three of them $(2 \mathrm{M} / 1 \mathrm{~F})$ also presented pinpoint lesions appearing as red, small papules of $4 \mathrm{~mm}$ diameter with mild scaling that were also biopsied. In six other patients, biopsies were taken in a
Table 1

Characteristics of the patients included in the study. F, female; M, male; N, uninvolved psoriatic skin, PP, early psoriasis lesion, PSO, psoriasis plaque.

\begin{tabular}{clllll}
\hline Patient & Age & Gender & PASI score & Site & Types of lesions \\
\hline 1 & 34 & F & 12 & Abdomen & N, PSO \\
2 & 31 & M & 14 & Arm & N, PSO \\
3 & 68 & M & 19 & Buttock & N, PSO \\
4 & 50 & M & 30 & Leg & N, PSO \\
5 & 28 & M & 5 & Leg & N, PSO \\
6 & 46 & M & 13 & Buttock & N, PSO \\
7 & 43 & M & 10 & Arm & N, PSO \\
8 & 32 & M & 22 & Abdomen & N, PP, PSO \\
9 & 70 & F & 18 & Back & N, PP, PSO \\
10 & 28 & M & 28 & Back & N, PP, PSO \\
11 & 42 & M & 11 & Arm & N, PP \\
12 & 40 & M & 14 & Back & N, PP \\
13 & 25 & F & 11 & Abdomen & N, PP \\
14 & 59 & F & 17 & Arm & N, PP \\
15 & 25 & M & 19 & Arm & N, PP \\
16 & 38 & F & 15 & Buttock & N, PP \\
17 & 47 & M & 11 & Arm & N, PSO \\
\hline
\end{tabular}

pinpoint lesion (PP) and in the uninvolved skin at distance from the lesion (mean age of all patients in the PP group: 40 years old, $4 \mathrm{~F} /$ 5M, mean PASI score: 17.2). An example of a pinpoint lesion is shown in Fig. 1.

Most of the biopsies were cut in two parts, one being fixed in 3\% formalin and embedded in paraffin for immunohistochemistry and the second being embedded in OCT and kept at $-80^{\circ} \mathrm{C}$.

This study was approved by the Ethics Committee of the University Hospital of Liège. The patients gave their written informed consent to participate to the study and the Declaration of Helsinki protocols were followed.

\subsection{RNA isolation and RT-PCR}

Fifty $\mu \mathrm{m}$ sections from OCT-embedded biopsies were collected and the surface of each biopsy was measured after digitization of a section with the Axiovert 25 Zeiss microscope coupled to a CCD camera (Carl Zeiss Vision GmbH). After washing OCT, RNA extraction was performed by phenol/chloroform extraction followed by glass-fiber filter purification (Ribopure Kit, Ambion,

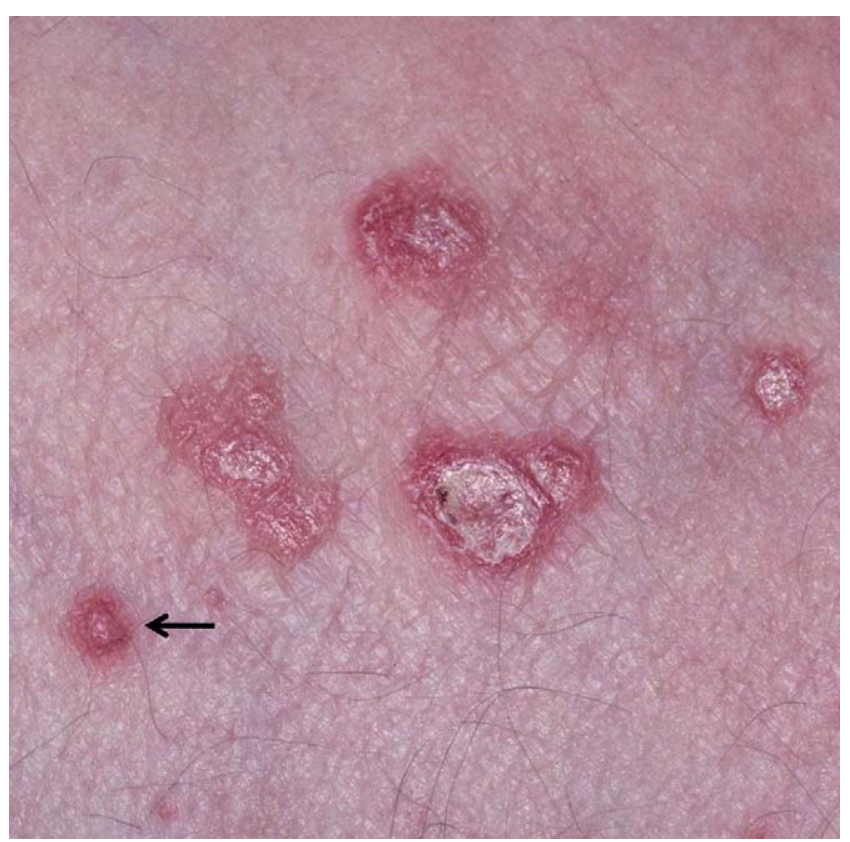

Fig. 1. Pinpoint lesion. This photograph shows psoriasis lesions including a pinpoint lesion (arrow). 
Austin, Texas, USA). RT-PCR were performed to measure 28S rRNA, K16, VEGF-A, VEGF-C, VEGFR2, VEGFR3, NRP-1, NRP-2a, prox-1, PIGF-1 and -2 mRNA, using a Superscript II Reverse Transcriptase (Invitrogen, Merelbeke, Belgium) and Takara Taq polymerase (Biomedical group, Shiga, Japan). RT-PCR amplifications were performed in an automated thermal cycler (GeneAmp PCR System 9700, Applied Biosystems, Foster City, USA) with different pairs of primers as previously described $[10,27,28]$. For VEGF-A and PIGF, forward and reverse primers were chosen in regions surrounding the alternatively skipped sequences, allowing the discrimination of the various splice variants on the basis of the size of their amplification product [29]. The amplification products were separated by polyacrylamide gel electrophoresis, stained (Gelstar, Cambrex, Rockland, USA) and quantified (Fluor-S Multilmager, Biorad, Hercules, CA, USA).

The total volume of tissue used for extracting RNA was evaluated by multiplying the number of sections by their surface and thickness. Quantification of the recorded signals was expressed in arbitrary units either per unit volume of tissue or per unit of $28 \mathrm{~S}$ ribosomal RNA, when appropriate.

\subsection{Histology}

Immunohistochemistry was performed as previously described [10] for Ki-67 (Clone MIB-1, DakoCytomation, Glostrup, Denmark), von Willebrand factor (Dakocytomation) and D2-40 (Proteogenix, Illrich Cedex, France). We also performed immunohistochemistry with anti-VEGFR3 (Clone 9D9F9, Millipore, Billerica, MA, USA) followed by incubation with a goat anti-mouse antibody coupled to biotin and with streptavidin coupled to peroxidase (both from Dakocytomation). Peroxidase activity was revealed with 3-amino9-ethylcarbazole.

\subsection{Image analysis}

\subsubsection{Visual image quantification}

Acanthosis score, papillomatosis score and Ki-67 labelling index expressed as the number of Ki-67-positive cells in percentage of the total number of basal and suprabasal cells taken as $100 \%$ in the epidermis were evaluated as previously described [10]. The number of cells positive for Ki-67 in the dermis (inside and outside vascular structures, consisting, respectively in endothelial and inflammatory cells) was counted in both the superficial dermis and the papillaries and normalised by stratum corneum length. The number of lymphatic vessels positive for VEGFR3 in the superficial dermis was counted for each group of biopsies and normalised by stratum corneum length.

\subsection{Semi-automatic images quantification}

Images of sections were observed and digitized as already described [10]. An algorithm for image processing and measurements was implemented, as previously described [10]. The following parameters were measured for blood and lymphatic vessels: (a) the average individual surface, (b) the average distance to the basement membrane and (c) the average total surface per area of superficial dermis (dermis between the superficial vascular plexus and the dermo-epidermal basement membrane).

\subsection{Statistical evaluation}

Results were expressed as means \pm standard deviations (SD). Statistical analysis was performed using the Mann-Whitney tests to compare the histological parameters of the groups. Unpaired T-test Welch corrected was applied for the RT-PCR results as normality tests were passed (method of Kolmogorov and Smirnov). Kruskal-Wallis tests were also performed to compare the three groups. Results were considered to be significant at the $5 \%$ confidence level $(p<0.05)$.

\section{Results}

\subsection{General tissue morphology}

Acanthosis and papillomatosis scores were evaluated for the three types of lesions (N, PP and PSO) and were significantly increased in PP as compared to N (Fig. 2) and further higher in PSO. The Ki-67 labeling index was similarly increased by a factor 2 in PP and PSO epidermis as compared to $\mathrm{N}$.

As seen in Fig. 2, the PP selected in our study are nascent psoriatic lesions that already display some degree of dermal and epidermal changes. The papillomatosis and acanthosis scores in PP lie between $\mathrm{N}$ and PSO indicating that these PP are evolving lesions towards plaques. We therefore used them as a model to examine the course of blood and lymphatic vascular expansion and related transcriptional markers.

\subsection{Blood vessel morphometry}

Proliferating (stained for Ki-67) blood endothelial cells were observed in the superficial dermis of PP and PSO (Fig. 3). Their number, normalised per unit of stratum corneum length, did not significantly differ between PSO and PP. They were most often found in the vertical limb of dermal vessels as previously described [30] but also in vessels beneath the basement membrane or in the superficial dermal plexus.

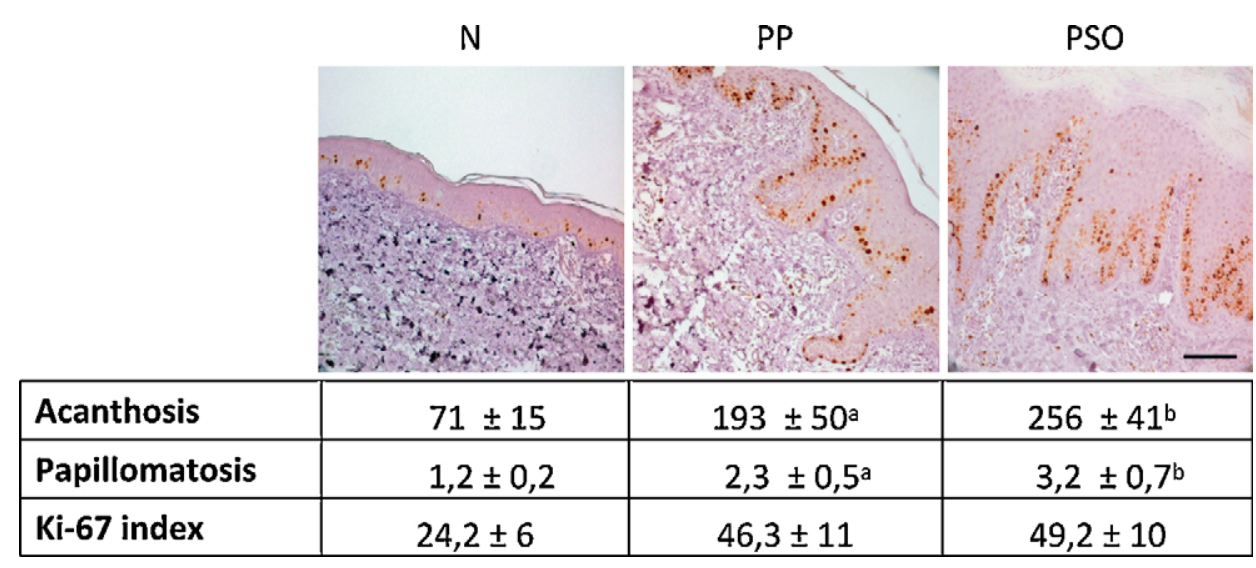

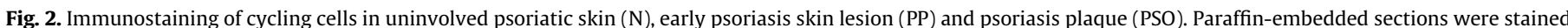

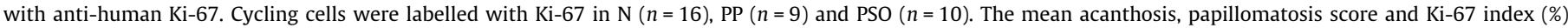
calculated as the ratio of labelled cells/total basal and epibasal cells are indicated in the table below. Scale bar $=100 \mu \mathrm{m}$. $p<0.01$ a PP vs. N; $\mathrm{b}$ PSO vs. PP. 

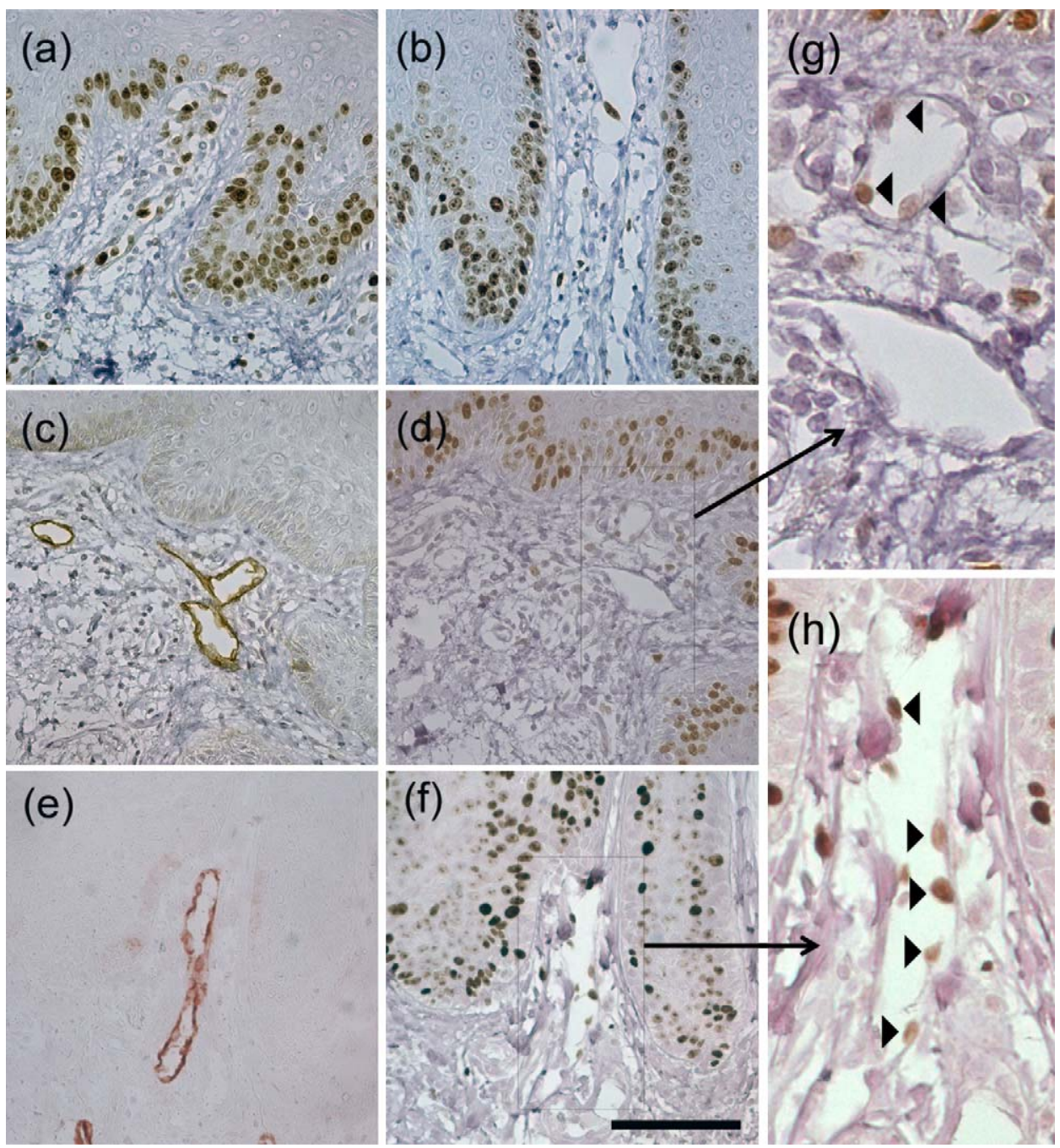

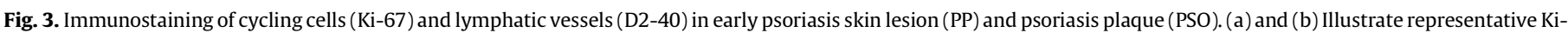

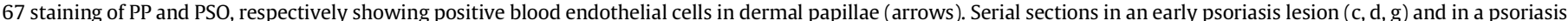

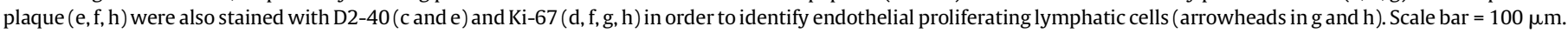

The average surface of individual blood vessels, identified by their positivity for von Willebrand factor (Fig. 4), in PP $\left(385 \pm 214 \mu \mathrm{m}^{2}\right.$, mean $\left.\pm \mathrm{SD}\right)$ was significantly higher than in $\mathrm{N}$ $\left(201 \pm 65 \mu \mathrm{m}^{2}\right)$ but was however significantly smaller than in PSO $\left(588 \pm 250 \mu \mathrm{m}^{2}\right)$. The number of blood vessel sections per unit surface was $110 \pm 46$ in $\mathrm{N}$ and almost doubled in PP $(200 \pm 55$, $p=0.0001)$. It significantly decreased in PSO $(145 \pm 24, p=0.004)$. The average total surface of blood vessels represented $1.25 \pm 0.9 \%$ of the superficial dermis area in N. It was significantly augmented in PSO $(4.2 \pm 1.7 \%)$, as we previously reported [10], and was found increased to a similar extent in early PP $(4.3 \pm 3.0 \%)$.

These data suggest that the percentage of dermis occupied by blood vessels is similar in PP and PSO but that vascular network evolves toward an enlargement of blood vessels in PSO.

We could not detect any significant difference in the mean distance of blood vessels to the basement membrane between the groups (N: $84 \pm 28 \mu \mathrm{m}$, PP: $68 \pm 19 \mu \mathrm{m}$, PSO: $81 \pm 19 \mu \mathrm{m}$ ).

\subsection{Lymphatic vessels morphometry}

Lymphatic vessels were detected as positive for podoplanin, a transmembrane glycoprotein recognised by anti-D2-40 antibody (Fig. 4) and expressed by a variety of human cell types, including lymphatic endothelium, but not by blood endothelial vessels $[31,32]$. The average size of lymphatic vessels sections was similar in $\mathrm{PP}\left(267 \pm 117 \mu \mathrm{m}^{2}\right)$ and in $\mathrm{N}\left(275 \pm 225 \mu \mathrm{m}^{2}\right)$ while it was higher in PSO $\left(427 \pm 98 \mu \mathrm{m}^{2}\right)$. The percentage of superficial dermis area occupied by lymphatic vessels was significantly higher in PP $(1.4 \pm 0.7 \%)$ than in $\mathrm{N}(0.36 \pm 0.30 \%)$ and further higher in PSO $(2.1 \pm 0.6 \%)$ (Fig. 4). As shown in Fig. 3, Ki-67-positive proliferating cells were observed lining the lymphatic vessels in PP as well as in PSO.

We previously described that lymphatics are closer to the basement membrane in PSO as compared to N [10]. A similar finding was observed in the series of psoriatic lesions analysed in this study (N: $96 \pm 53 \mu \mathrm{m}$; PSO: $66 \pm 16 \mu \mathrm{m}$ ). The PP depicted here had also a reduced mean lymphatic distance to basement membrane (PP: $65 \pm 23 \mu \mathrm{m}$ ) as compared to $\mathrm{N}$ although at the limit of the statistical significance $(p=0.055)$.

These morphometric data indicate that blood vasculature has already expanded in PP to an extent similar to that observed in PSO. By contrast, the lymphatic vascular network in PP is in a remodelling and expanding process suggesting that lymphatic vessel development is posterior to the blood vessels expansion in psoriasis.

\subsection{Selected transcriptomic analysis}

The morphometric analyses described above clearly show epidermal alterations in PP such as increased acanthosis and 


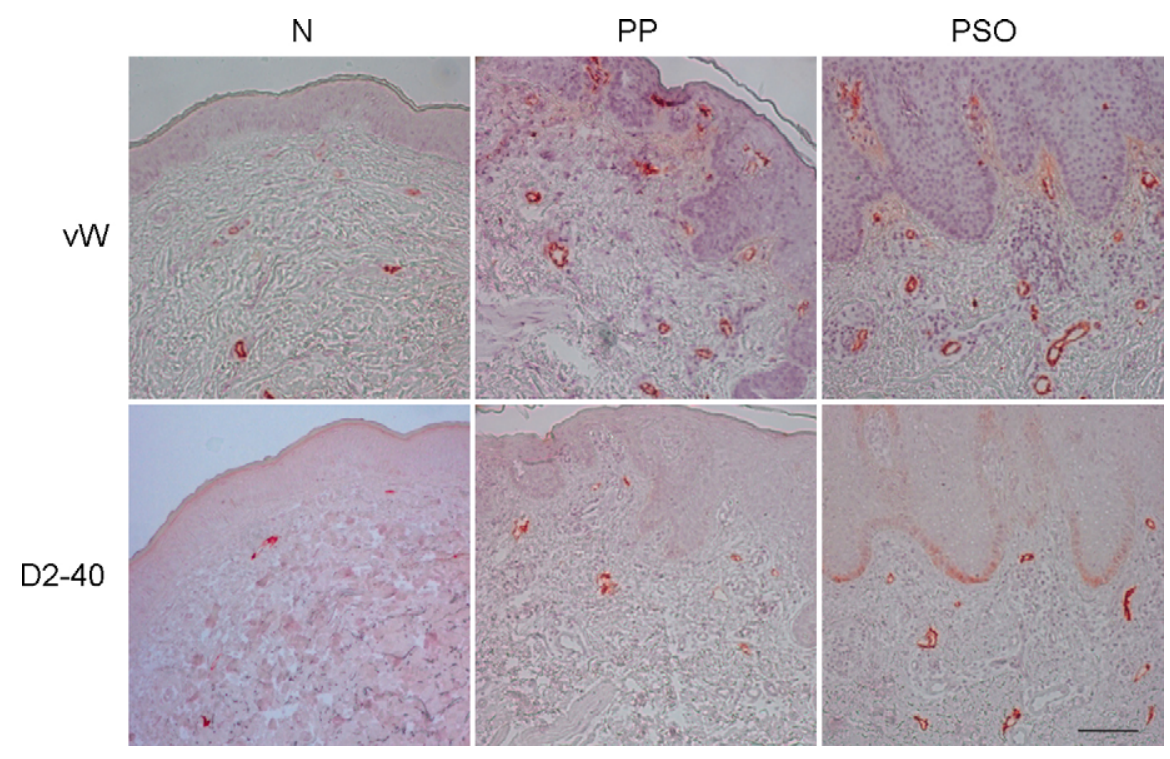

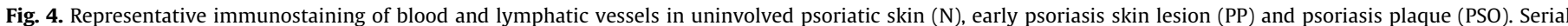

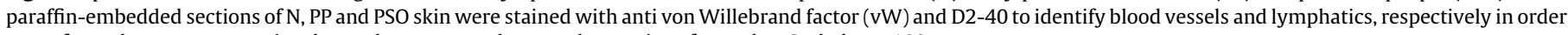
to perform the computer-assisted morphometry on the complete series of samples. Scale bar $=100 \mu \mathrm{m}$.

papillomatosis scores and enhanced keratinocyte proliferation as compared to non-lesional skin. Inflammatory cells infiltrate has also been described in these early lesions [26]. The contribution of epidermal and inflammatory cells in the total RNA extracted from such lesions for transcriptomic analyses is significantly larger and prevents a correct interpretation of the data when comparing $\mathrm{N}$ and PP or PSO but also when analyzing genes originating from dermis. To overcome this bias, the expression of a panel of selected genes was compared in $\mathrm{N}$ and $\mathrm{PP}$ on the basis of a unit volume of tissue. This was made possible by extracting RNA from sections of frozen tissues in OCT of known thickness and area. Psoriatic lesions were similarly handled. For genes known to be expressed in epidermis and inflammatory infiltrate such as K16 and VEGF-A, data in PP and PSO were also calculated on the basis of 28S rRNA, enabling us to normalise the expression of those epidermal genes on a per cell basis.

Table 2

Expression of some general, angiogenesis and lymphangiogenesis markers in uninvolved skin of psoriasis patients $(\mathrm{N})$, early pinpoint lesions (PP) and psoriasis plaques (PSO).

\begin{tabular}{lccc}
\hline mRNA & $\mathrm{N}(n=15)$ & $\mathrm{PP}(n=8)$ & PSO $(n=9)$ \\
\hline 28S & $6.3 \pm 5.6^{\mathrm{a}}$ & $20.2 \pm 9.7^{\mathrm{b}}$ & $22.9 \pm 19.9^{\mathrm{b}}$ \\
Keratin 16 & $14.7 \pm 12.2$ & $512 \pm 319^{\mathrm{b}}$ & $997 \pm 846^{\mathrm{b}}$ \\
Angiogenesis markers & & & \\
VEGF-A 121 & $6.0 \pm 3.8$ & $22.0 \pm 7.9^{\mathrm{b}}$ & $21.7 \pm 16.1^{\mathrm{b}}$ \\
VEGF-A 165 & $2.7 \pm 1.9$ & $11.0 \pm 4.6^{\mathrm{b}}$ & $8.2 \pm 4.8^{\mathrm{b}}$ \\
VEGF-A 189 & $0.5 \pm 0.4$ & $4.6 \pm 2.4^{\mathrm{b}}$ & $2.2 \pm 1.6^{\mathrm{b}}$ \\
VEGFR2 & $14.1 \pm 9.3$ & $35.9 \pm 26.6^{\mathrm{b}}$ & $39.1 \pm 26.8^{\mathrm{b}}$ \\
NRP-1 & $7.1 \pm 5.6$ & $19.6 \pm 9.3^{\mathrm{b}}$ & $21.1 \pm 15.1^{\mathrm{b}}$ \\
PIGF-1 & $12.0 \pm 13.4$ & $52.7 \pm 40.3$ & $48.9 \pm 38.6^{\mathrm{b}}$ \\
PIGF-2 & $27.7 \pm 19.6$ & $105 \pm 31^{\mathrm{b}}$ & $125 \pm 112^{\mathrm{b}}$ \\
Lymphangiogenesis markers & & \\
VEGF-C & $15.7 \pm 11.0$ & $39.8 \pm 8.4^{\mathrm{b}}$ & $44.4 \pm 23.9^{\mathrm{b}}$ \\
VEGFR3 & $7.0 \pm 6.8$ & $10.5 \pm 3.8^{\mathrm{b}}$ & $16.7 \pm 7.8^{\mathrm{b}}$ \\
NRP-2a & $6.7 \pm 6.5$ & $21.3 \pm 9.9^{\mathrm{b}}$ & $25.1 \pm 18.8$ \\
Prox-1 & $5.8 \pm 6.6$ & $12 \pm 10.0$ & $17.5 \pm 11.3^{\mathrm{b}}$ \\
\hline
\end{tabular}

Results are expressed as arbitrary units by unit of tissue volume. VEGF, vascular endothelial growth factor; NRP, neuropilin; PIGF, placental growth factor.

a Mean \pm standard deviation.

b Significant statistical difference when compared to $N(p<0.05)$.

\section{5. $28 \mathrm{~S}$ rRNA and $\mathrm{K} 16$}

The expression of $28 \mathrm{~S}$ ribosomal RNA was evaluated in the three groups by unit of tissue volume (Table 2). As expected, it was clearly increased in PP as compared to N. Its mean level was however not further increased in PSO. Keratin 16, a marker of proliferating epidermal cells, was also overexpressed in PP and PSO when compared to $\mathrm{N}$. When expressed per unit of 28S rRNA (Table 3), K16 was significantly lower in PP than in PSO, consistent with milder epidermal changes in PP and higher papillomatosis and acanthosis scores in PSO.

\subsection{Angiogenesis growth factors, receptors and co-receptors}

\subsubsection{VEGF-A}

The expression of VEGF-A 121, 165 and 189 was significantly increased in PP and PSO (Table 2) as compared to N. It is noteworthy that although the total VEGF-A was similar in PP and PSO, the VEGF-A 189 mRNA calculated on a per cell basis (Table 3 ) was significantly overexpressed in PP as compared to PSO (Fig. 5). The proportion of the splice variant VEGF-A 189 mRNA was indeed higher in PP $(10.2 \pm 3.4 \%)$ as compared to $\mathrm{N}$ and PSO ( $5.3 \pm 2.8 \%$ and $6.8 \pm 2 \%$, respectively). This was further confirmed in the three patients biopsied in N, PP and PSO, who always had a higher proportion of VEGF-A 189 mRNA in PP as compared to PSO.

Table 3

Expression of keratin 16, total VEGF-A and proportion (\%) of the various isoforms of VEGF-A in early psoriasis lesions (PP) and psoriasis plaques (PSO).

\begin{tabular}{lcr}
\hline & PP $(n=8)$ & PSO $(n=9)$ \\
\hline Keratin 16 & $3.2 \pm 1.1^{\mathrm{a}, \mathrm{b}}$ & $5.2 \pm 1.8$ \\
Total VEGF-A & $2.9 \pm 1.4$ & $1.9 \pm 0.5$ \\
VEGF-A 121\% & $59.6 \pm 3.1^{\mathrm{a}}$ & $66.2 \pm 5.2$ \\
VEGF-A 165\% & $30.2 \pm 4.9$ & $26.9 \pm 4.5$ \\
VEGF-A 189\% & $10.2 \pm 3.4^{\mathrm{b}}$ & $6.8 \pm 2.1$ \\
\hline
\end{tabular}

Results are expressed in arbitrary units by unit of 28S rRNA and in mean percentage of each isoform of VEGF-A in PP and PSO. VEGF, vascular endothelial growth factor.

a Mean \pm standard deviation.

b Significant statistical difference between PP and PSO $(p<0.05)$ 


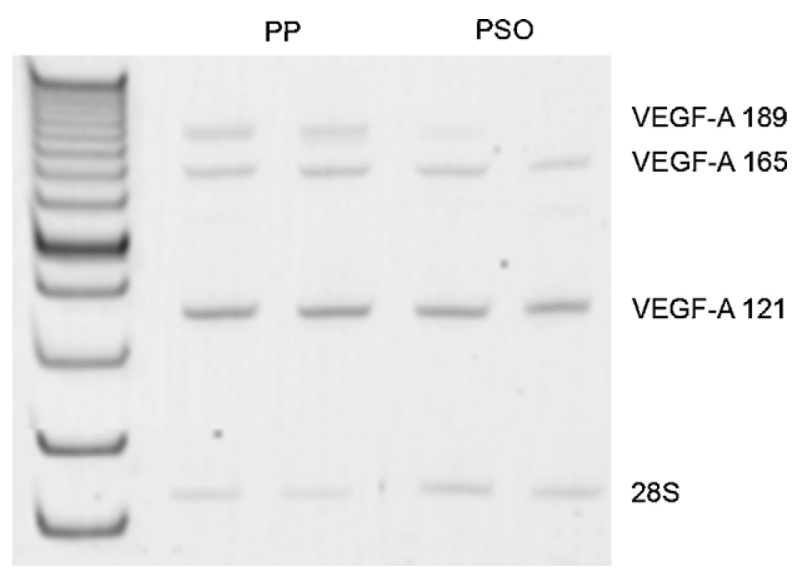

Fig. 5. Electrophoretic pattern of the amplified products of VEGF-A and $28 \mathrm{~S}$ genes. RNA was extracted from OCT-embedded punch biopsies of early psoriasis skin lesion (PP) and psoriasis plaque (PSO). Reverse transcription-polymerase chain reaction (RT-PCR) was performed in duplicates for $28 \mathrm{~S}$ and VEGF-A. The amplified products were separated by polyacrylamide gel electrophoresis, stained with ethidium bromide and quantified.

\subsection{VEGFR2, NRP-1, PlGF-1 and PlGF-2}

As compared to N, VEGFR2, NRP-1, PlGF-1 and -2 mRNA were increased in PP and in PSO (Table 2).

\subsection{Lymphangiogenesis markers}

As compared to N, VEGF-C and NRP-2a were overexpressed to a similar level in PP and PSO (Table 2). The expression of VEGFR3 per unit volume of tissue was increased in PSO when compared to $\mathrm{N}$ (Table 2) while PP showed intermediate values. This was confirmed at the protein level by histological staining of VEGFR3 and podoplanin in serial sections. VEGFR3-positive lymphatic vessels were almost absent in N skin (not shown) while PP indeed showed a number of VEGFR3-positive lymphatic vessels that further increased in PSO (Fig. 6). We want to stress out here that epidermal cells are negative for VEGFR3. The specific lymphangiogenesis marker prox-1 displayed an intermediate expression in PP between $\mathrm{N}$ and PSO (Table 2).

\section{Discussion}

In our study, significant morphological differences were found between early papular lesions (pinpoint, PP) and established psoriasis plaques (PSO). The first evident difference was a smaller acanthosis and papillomatosis score in PP. The percentage of Ki-67 labelled cells in the basal and epibasal layers was similar in the two groups suggesting that epidermal cells were proliferating at a similar rate, the epidermal compartment being however smaller in PP. The early psoriasis lesions that we studied here already comprise some epidermal changes in addition to the first dermal alterations described in the literature such as inflammatory infiltrates and vascular changes.

Compared to N, the area occupied by blood vessels in PP was already increased to a level similar to PSO. The vasculature pattern however evolved during the transition between early to established lesions by enlargement of blood vessels and a reduced density of vascular sections. On the contrary, the area occupied by lymphatic vessels is lower in PP than in PSO, but already increased as compared to $\mathrm{N}$. This is due to an increased lymphatic vessels density without any modification of their mean diameter as compared to $\mathrm{N}$. These data suggest that lymphatic vessels development is posterior to blood vascular network expansion in psoriasis. This is also supported by the expression of prox-1 and

\section{D2-40}
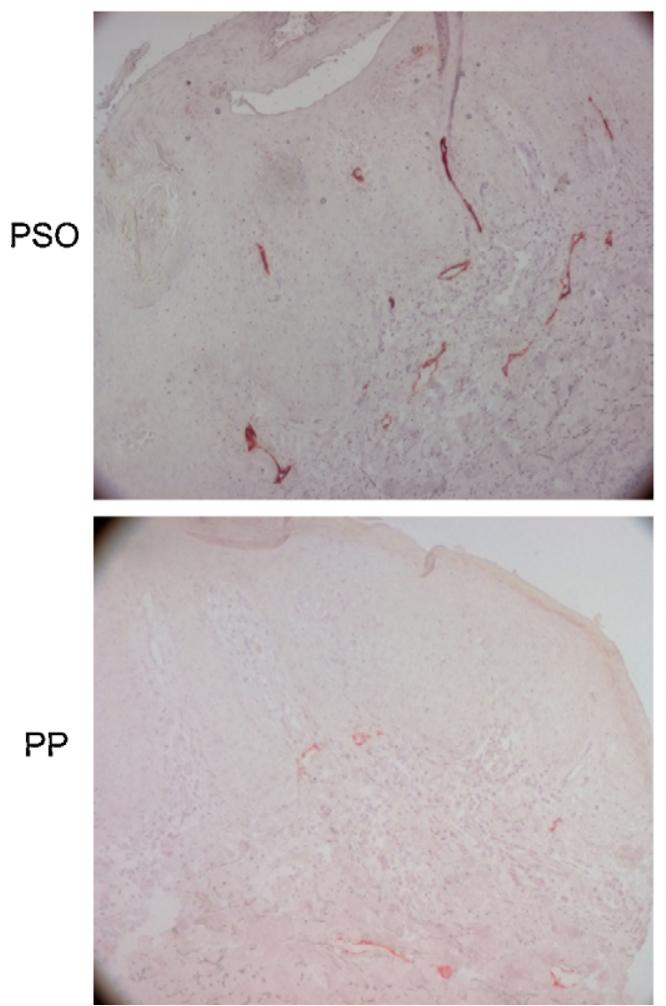

\section{VEGFR3}
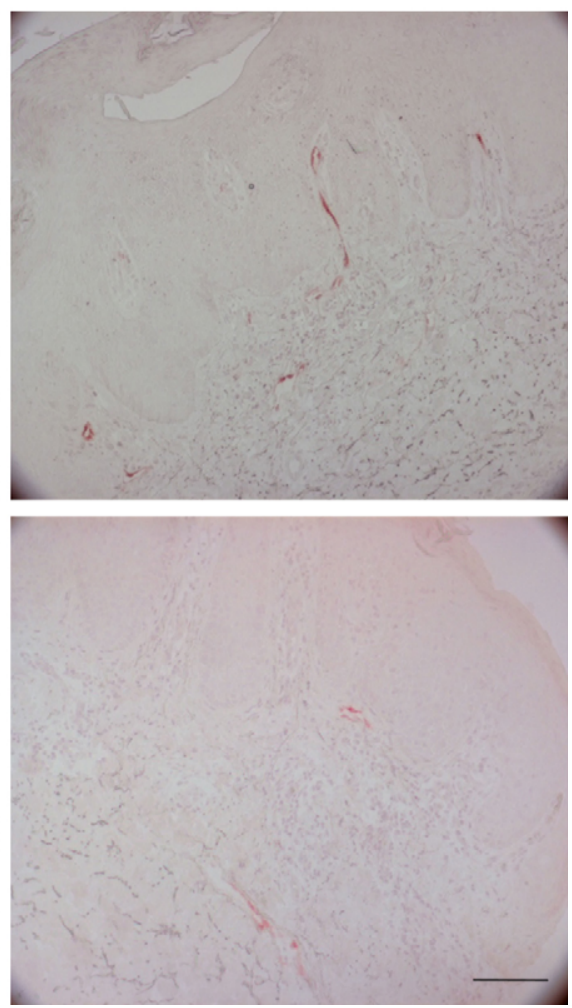

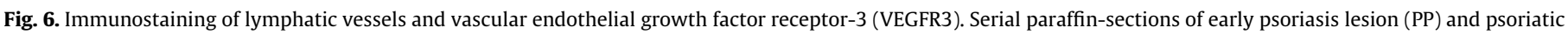
plaque (PSO) were stained with anti-human D2-40 (lymphatic vessels) and anti-human VEGFR3. Scale bar $=100 \mu \mathrm{m}$. 
VEGFR3, two markers of active lymphangiogenesis, which is intermediate between $\mathrm{N}$ and PSO while the expression of angiogenesis markers is similar in PP and PSO. This time sequence in human psoriasis progression can be related to lymphatic growth that also follows blood vascular expansion during mice development [19] and wound healing where it occurs about 1 week later [33].

In this study, we used the 9D9F9 clone mab to detect VEGFR3. While a positive VEGFR3 staining was observed in lymphatic vessels in the dermis, we did not observe the intense staining in psoriatic epidermis reported by other authors who used the MAB3491 [34]. To further investigate a potential expression of VEGFR3 by keratinocytes as reported by these authors, we performed RT-PCR on resting, proliferating and differentiating human keratinocytes in vitro with negative results (data not shown). Similarly, RT-PCR performed on microdissection LASER capture of normal epidermis failed to detect any VEGFR3 (data not shown). The data reported by the team of Alitalo [35] has demonstrated that MAB3491 recognises a non-specific antigen and that 9D9F9 is a more reliable antibody to investigate the expression of VEGFR3. We therefore conclude that VEGFR3 is not expressed in human normal or psoriatic epidermis.

Very few lymphatic vessels expressed the VEGFR3 protein in the uninvolved skin suggesting that, similarly to the findings in mice a few weeks after birth [36], lymphatic vasculature becomes independent of VEGFR3 ligands in adult non-regenerating healthy skin. VEGFR3-positive vessels are present in the dermal papillae of $\mathrm{PP}$ and further increased in PSO where lymphangiogenesis is more pronounced. The data presented here suggests that VEGFR3mediated signals are significant in the development and/or maintenance of lymphatic vessels in the psoriasis process. A role for VEGFR3 in psoriasis is also suggested by our previous studies showing an overexpression of VEGFR3 mRNA in uninvolved psoriasis skin compared to skin from healthy volunteers [10]. It is further supported by the association between psoriasis and two single nucleotide polymorphisms in the VEGFR3 gene as well as a higher serum level of VEGFR3 in psoriatic patients [37]. The parallel evolution in the expression of VEGFR3 and prox-1, a homeobox gene essential in its regulation, is a further argument supporting the activation of lymphangiogenesis in psoriasis.

Another significant finding in this study is the overexpression of the VEGF-A 189 mRNA isoform in early psoriasis lesions as compared to uninvolved skin and to psoriasis plaques. It has been postulated that the differential localization and receptor affinity of VEGF-A isoforms might influence the behaviour of the responding endothelium [38]. In vivo studies have suggested that heparinbinding VEGF-A isoforms, such as VEGF 189, provide spatially restricted stimulatory cues that polarize and thereby guide sprouting endothelial cells during vascular branch formation, while soluble forms lead to enlargement of lumen size [38]. The increased expression of VEGF-A 121 mRNA in the uninvolved skin of psoriasis patients compared to skin of healthy volunteers [10] and the observation of a transient elevated VEGF-A 189 level in early psoriasis lesions that we demonstrate here support the hypothesis of a sequential and coordinated action of the various VEGF isoforms leading to vascular expansion in inflammatory processes.

It is generally admitted that vascular expansion in psoriasis is secondary to vessel enlargement [39], elongation [40] and increased tortuosity instead of an actual sprouting angiogenesis. Our histological data suggest that the early psoriasis lesions, which present a higher VEGF-A 189/VEGF-A 121 transcripts ratio, comprise elongated and partly enlarged tortuous blood vessels. The subsequent increase in blood vessel size observed in psoriasis plaques compared to PP, is paralleled by a decrease in the VEGF-A 189/VEGF-A 121 transcripts balance. VEGF 189 is chemotactic for polymorphonuclear cells, probably through VEGFR1, and can be locally released by PMN, participating in this way in an autocrine amplification mechanism [41]. The numerous neutrophils already present in the initial phases of psoriasis could be a source of VEGF 189 and then enhance the inflammatory process [41].

VEGF-C mRNA expression did not differ in PP and PSO and was increased as compared to $\mathrm{N}$. Other VEGFR3 ligands could be implicated in the lymphatic vascular expansion. Some previous reports have already implicated VEGF-A as a potent trigger of lymphangiogenesis but it is still unclear if this effect is direct, through VEGFR2 as described in tissue repair [33], or indirect via up-regulation of lymphangiogenic factors.

VEGF-A as well as VEGF-C are cytokines that can constitute a link between inflammation and vascular expansion in psoriasis. Both of these molecules are secreted by inflammatory cells as activated macrophages and induce the recruitment of some immune cells expressing the corresponding VEGFR, in addition to their (lymph)angiogenic role [42-44]. This bridge between angiogenesis, lymphangiogenesis, which are respectively the growth of new blood and lymphatic vessels from pre-existing ones, and inflammation underline the importance of vascular remodelling in psoriasis and the potential therapeutic perspectives by inhibiting these processes. Furthermore, a role for lymphatic vessels in modelling immunologic responses has recently been described $[45,46]$, supporting the importance to better understand their function in psoriasis. Use of anti-(lymph)angiogenic agents in topical application would likely avoid unwanted side effects as observed in systemic treatments.

This work studying lesions transitional between normal skin and psoriasis plaques emphasises a probable role for VEGF-A 189 during the development of the peculiar vascular architecture in psoriasis. It also adds evidence for a role of VEGFR3 in the expansion of the lymphatic network in psoriasis plaque and shows that this expansion seems to follow that of blood vessels.

\section{Acknowledgments}

A.H. is a Research Fellow, C.D. and A.C. are Senior Research Associate of the Belgian National Scientific Research Fund (FNRS). We thank Antoine Heyeres and Marie-Jeanne Nix for their skilful technical assistance, Annick Rausin and Jocelyne Renson for their kind participation in the recruitment of volunteers. Yves Poumay is gratefully acknowledged for the kind gift of keratinocytes mRNA. This work was supported by a grant from the Belgian Fund for Scientific Medical Research (FRSM), by the "Region Wallonne" (Neoangio) and by the "Fonds Léon Frédéricq" of the University of Liege.

\section{References}

[1] Griffiths CE, Barker JN. Pathogenesis and clinical features of psoriasis. Lancet 2007;370:263-71.

[2] Braverman IM, Yen A. Ultrastructure of the capillary loops in the dermal papillae of psoriasis. J Invest Dermatol 1977;68:53-60.

[3] de la Brassinne M, Lachapelle JM. 3H-thymidine labelling of epidermis and dermal infiltrate in psoriatic erythroderma. Acta Derm Venereol 1975;55: 171-4.

[4] Creamer D, Sullivan D, Bicknell R, Barker J. Angiogenesis in psoriasis. Angiogenesis 2002;5:231-6.

[5] Lowe PM, Lee ML, Jackson CJ, To SS, Cooper AJ, Schrieber L. The endothelium in psoriasis. Br J Dermatol 1995;132:497-505.

[6] Adams RH, Alitalo K. Molecular regulation of angiogenesis and lymphangiogenesis. Nat Rev Mol Cell Biol 2007;8:464-78.

[7] Detmar M, Brown LF, Claffey KP, Yeo KT, Kocher O, Jackman RW, et al. Overexpression of vascular permeability factor/vascular endothelial growth factor and its receptors in psoriasis. J Exp Med 1994;180:1141-6.

[8] Ferrara N, Gerber HP, LeCouter J. The biology of VEGF and its receptors. Nat Med 2003;9:669-76.

[9] Mineur P, Colige AC, Deroanne CF, Dubail J, Kesteloot F, Habraken Y, et al. Newly identified biologically active and proteolysis-resistant VEGF-A isoform VEGF111 is induced by genotoxic agents. J Cell Biol 2007;179:1261-73. 
[10] Henno A, Blacher S, Lambert C, Colige A, Seidel L, Noel A, et al. Altered expression of angiogenesis and lymphangiogenesis markers in the uninvolved skin of plaque-type psoriasis. Br J Dermatol 2009;160:581-90.

[11] Cianfarani F, Zambruno G, Brogelli L, Sera F, Lacal PM, Pesce M, et al. Placenta growth factor in diabetic wound healing: altered expression and therapeutic potential. Am J Pathol 2006;169:1167-82.

[12] Oura H, Bertoncini J, Velasco P, Brown LF, Carmeliet P, Detmar M. A critical role of placental growth factor in the induction of inflammation and edema formation. Blood 2003;101:560-7.

[13] Cao Y, Ji WR, Qi P, Rosin A. Placenta growth factor: identification and characterization of a novel isoform generated by RNA alternative splicing. Biochem Biophys Res Commun 1997;235:493-8.

[14] Yang W, Ahn H, Hinrichs M, Torry RJ, Torry DS. Evidence of a novel isoform of placenta growth factor (PIGF-4) expressed in human trophoblast and endothelial cells. J Reprod Immunol 2003;60:53-60.

[15] Oliver G, Detmar M. The rediscovery of the lymphatic system: old and new insights into the development and biological function of the lymphatic vasculature. Genes Dev 2002;16:773-83.

[16] Karkkainen MJ, Haiko P, Sainio K, Partanen J, Taipale J, Petrova TV, et al. Vascular endothelial growth factor $C$ is required for sprouting of the first lymphatic vessels from embryonic veins. Nat Immunol 2004;5:74-80.

[17] Partanen TA, Arola J, Saaristo A, Jussila L, Ora A, Miettinen M, et al. VEGF-C and VEGF-D expression in neuroendocrine cells and their receptor. VEGFR-3, in fenestrated blood vessels in human tissues. FASEB J 2000;14:2087-96.

[18] Favier B, Alam A, Barron P, Bonnin J, Laboudie P, Fons P, et al. Neuropilin-2 interacts with VEGFR-2 and VEGFR-3 and promotes human endothelial cell survival and migration. Blood 2006;108:1243-50.

[19] Saharinen P, Tammela T, Karkkainen MJ, Alitalo K. Lymphatic vasculature: development, molecular regulation and role in tumor metastasis and inflammation. Trends Immunol 2004;25:387-95.

[20] Hong YK, Harvey N, Noh YH, Schacht V, Hirakawa S, Detmar M, et al. Prox1 is a master control gene in the program specifying lymphatic endothelial cell fate. Dev Dyn 2002;225:351-7.

[21] Kunstfeld R, Hirakawa S, Hong YK, Schacht V, Lange-Asschenfeldt B, Velasco P, et al. Induction of cutaneous delayed-type hypersensitivity reactions in VEGFA transgenic mice results in chronic skin inflammation associated with persistent lymphatic hyperplasia. Blood 2004;104:1048-57.

[22] Henno A, Lapiere CM, Nusgens BV, de la Brassinne M. Lymphatic vasculature: dermatological implications of emerging concepts. Ann Dermatol Venereo 2008;135:704-9.

[23] Christophers E, Parzefall R, Braun-Falco O. Initial events in psoriasis: quantitative assessment. Br J Dermatol 1973;89:327-34.

[24] Braun-Falco O, Christophers E. Structural aspects of initial psoriatic lesions. Arch Dermatol Forsch 1974:251:95-110.

[25] Braun-Falco O, Schmoeckel C. The dermal inflammatory reaction in initial psoriatic lesions. Arch Dermatol Res 1977;258:9-16.

[26] Chowaniec O, Jablonska S, Beutner EH, Proniewska M, Jarzabek-Chorzelska M, Rzesa G. Earliest clinical and histological changes in psoriasis. Dermatologica 1981;163:42-51

[27] Nusgens BV, Humbert P, Rougier A, Colige AC, Haftek M, Lambert CA, et al. Topically applied vitamin $C$ enhances the mRNA level of collagens I and III their processing enzymes and tissue inhibitor of matrix metalloproteinase 1 in the human dermis. J Invest Dermatol 2001;116:853-9.

[28] Hajitou A, Sounni NE, Devy L, Grignet-Debrus C, Lewalle JM, Li H, et al. Downregulation of vascular endothelial growth factor by tissue inhibitor of metalloproteinase-2: effect on in vivo mammary tumor growth and angiogenesis. Cancer Res 2001;61:3450-7.
[29] Deroanne CF, Bonjean K, Servotte S, Devy L, Colige A, Clausse N, et al. Histone deacetylases inhibitors as anti-angiogenic agents altering vascular endothelial growth factor signaling. Oncogene 2002;21:427-36.

[30] Creamer D, Allen MH, Sousa A, Poston R, Barker JN. Localization of endothelial proliferation and microvascular expansion in active plaque psoriasis. $\mathrm{Br} \mathrm{J}$ Dermatol 1997;136:859-65.

[31] Halin C, Detmar M. Chapter 1. Inflammation, angiogenesis, and lymphangiogenesis. Methods Enzymol 2008;445:1-25.

[32] Schacht V, Dadras SS, Johnson LA, Jackson DG, Hong YK, Detmar M. Upregulation of the lymphatic marker podoplanin, a mucin-type transmembrane glycoprotein, in human squamous cell carcinomas and germ cell tumors. Am J Pathol 2005;166:913-21.

[33] Hong YK, Lange-Asschenfeldt B, Velasco P, Hirakawa S, Kunstfeld R, Brown LF, et al. VEGF-A promotes tissue repair-associated lymphatic vessel formation via VEGFR-2 and the alpha1beta1 and alpha2beta1 integrins. FASEB J 2004:18:1111-3.

[34] Man XY, Yang XH, Cai SQ, Yao YG, Zheng M. Immunolocalization and expression of vascular endothelial growth factor receptors (VEGFRs) and neuropilins (NRPs) on keratinocytes in human epidermis. Mol Med 2006;12:127-36.

[35] Petrova TV, Bono P, Holnthoner W, Chesnes J, Pytowski B, Sihto H, et al. VEGFR3 expression is restricted to blood and lymphatic vessels in solid tumors. Cancer Cell 2008;13:554-6.

[36] Karpanen T, Wirzenius M, Makinen T, Veikkola T, Haisma HJ, Achen MG, et al. Lymphangiogenic growth factor responsiveness is modulated by postnatal lymphatic vessel maturation. Am J Pathol 2006;169:708-18.

[37] Lee JH, Cho EY, Namkung JH, Kim E, Kim S, Shin ES, et al. Single-nucleotide polymorphisms and haplotypes in the VEGF receptor 3 gene and the haplotype GC in the VEGFA gene are associated with psoriasis in Koreans. J Invest Dermatol 2008;128:1599-603.

[38] Ruhrberg C, Gerhardt H, Golding M, Watson R, Ioannidou S, Fujisawa H, et al. Spatially restricted patterning cues provided by heparin-binding VEGF-A control blood vessel branching morphogenesis. Genes Dev 2002;16:2684-98.

[39] Carmeliet P. Mechanisms of angiogenesis and arteriogenesis. Nat Med 2000;6:389-95.

[40] Braverman IM, Sibley J. Role of the microcirculation in the treatment and pathogenesis of psoriasis. J Invest Dermatol 1982;78:12-7.

[41] Ancelin M, Chollet-Martin S, Herve MA, Legrand C, El Benna J, Perrot-Applanat M. Vascular endothelial growth factor VEGF189 induces human neutrophil chemotaxis in extravascular tissue via an autocrine amplification mechanism. Lab Invest 2004;84:502-12.

[42] Watari K, Nakao S, Fotovati A, Basaki Y, Hosoi F, Bereczky B, et al. Role of macrophages in inflammatory lymphangiogenesis: enhanced production of vascular endothelial growth factor C and D through NF-kappaB activation. Biochem Biophys Res Commun 2008;377:826-31.

[43] Hamrah P, Chen L, Zhang Q, Dana MR. Novel expression of vascular endothelial growth factor receptor (VEGFR)-3 and VEGF-C on corneal dendritic cells. Am J Pathol 2003;163:57-68.

[44] Detmar M, Brown LF, Schon MP, Elicker BM, Velasco P, Richard L, et al. Increased microvascular density and enhanced leukocyte rolling and adhesion in the skin of VEGF transgenic mice. J Invest Dermatol 1998;111:1-6.

[45] Teoh D, Johnson LA, Hanke T, McMichael AJ, Jackson DG. Blocking development of a CD8+ $\mathrm{T}$ cell response by targeting lymphatic recruitment of APC. J Immunol 2009;182:2425-31.

[46] Kataru RP, Jung K, Jang C, Yang H, Schwendener RA, Baik JE, et al. Critical role of $\mathrm{CD} 11 \mathrm{~b}+$ macrophages and VEGF in inflammatory lymphangiogenesis, antigen clearance, and inflammation resolution. Blood 2009;113:5650-9. 